ALEA, Lat. Am. J. Probab. Math. Stat. 16, 957-976 (2019)

DOI: 10.30757/ALEA.v16-34

\title{
Fast-reaction limit for Glauber-Kawasaki dynamics with two components
}

\author{
A. De Masi, T. Funaki, E. Presutti and M. E. Vares \\ Dipartimento di Ingegneria e Scienze dell'Informazione e Matematica \\ Università degli studi dell'Aquila, L'Aquila, 67100 Italy \\ E-mail address: demasi@univaq.it \\ Department of Mathematics, School of Fundamental Science and Engineering, \\ Waseda University, 3-4-1 Okubo, Shinjuku-ku, Tokyo 169-8555, Japan. \\ E-mail address: funaki@ms.u-tokyo.ac.jp \\ GSSI, viale F. Crispi 7, 67100 L'Aquila, Italy \\ E-mail address: errico.presutti@gmail.com \\ Instituto de Matemática. Universidade Federal do Rio de Janeiro, \\ Centro de Tecnologia - Bloco C, Av. Athos da Silveira Ramos, 149, \\ Ilha do Fundão, 21941-909, Rio de Janeiro, RJ, Brazil \\ E-mail address: eulalia@im.ufrj.br
}

\begin{abstract}
We consider the Kawasaki dynamics of two types of particles under a killing effect on a $d$-dimensional square lattice. Particles move with possibly different jump rates depending on their types. The killing effect acts when particles of different types meet at the same site. We show the existence of a limit under the diffusive space-time scaling and suitably growing killing rate: segregation of distinct types of particles does occur, and the evolution of the interface between the two distinct species is governed by the two-phase Stefan problem. We apply the relative entropy method and combine it with some PDE techniques.
\end{abstract}

\section{Introduction}

The study of the fast-reaction limit in reaction diffusion systems goes back more than 20 years. The motivation of this study comes from population dynamics Dancer et al. (1999), Crooks et al. (2004), Iida et al. (2017), mass-action kinetics chemistry Daus et al. (2017) and others. Consider the system consisted of two types of species, say $A$ and $B$, and assume each of them moves by diffusion with rates $d_{1}$ and $d_{2}$, respectively. When distinct species meet, they kill each other with high

Received by the editors March 21th, 2019; accepted July 1st, 2019.

2010 Mathematics Subject Classification. 60K35, 35K55, 35K57, 35R35, 80A22.

Key words and phrases. Hydrodynamical limit, relative entropy method, fast reaction limit, free boundary problem, singular limit problem. 
rate $K$. This problem is formulated in PDE terminology as the system of equations for densities $u_{1}(t, r)$ and $u_{2}(t, r)$ of species $A$ and $B$, respectively, written as

$$
\partial_{t} u_{i}(t)=d_{i} \Delta u_{i}(t)-K u_{1}(t) u_{2}(t), \quad i=1,2 .
$$

Several papers, including those cited above, studied the limit as $K \rightarrow \infty$ of the solutions $u_{i}(t, r)$ of the system (1.1) or its extensions, that is, the limit as the killing rate of distinct species gets large. This is called the fast-reaction limit. It is known that the segregation of two species occurs in the limit and the interface separating two distinct species evolves according to the two-phase Stefan free boundary problem.

In the present paper, we formulate the problem at the original level of species, i.e., at the underlying microscopic level, and model it as a system with two distinct types of particles. Under a diffusive space-time scaling combined with the limit as $K \rightarrow \infty$ taken properly, we prove that the segregation of species occurs at macroscopic level and derive the Stefan problem directly from our microscopic system.

The proof is divided into two parts and given as a combination of the techniques of the hydrodynamic limit and the fast-reaction limit. In the first part, which is probabilistic, we consider the relative entropy of the real system with respect to the local equilibria defined as a product measure with mean changing in space and time chosen according to the discretized hydrodynamic equation, which is a discrete version of (1.1). Then, we show that the relative entropy behaves as a small order of the total volume of the system. This proves that the macroscopic density profile of the system is close to the solution of the discretized hydrodynamic equation. We take product measures as local equilibria, since those with constant means are global equilibria of the Kawasaki dynamics. In the second part of the paper, we apply PDE results to analyze the discrete equation and derive the Stefan problem from it.

1.1. Model. Let $\mathbb{T}_{N}^{d}:=(\mathbb{Z} / N \mathbb{Z})^{d} \equiv\{1,2, \ldots, N\}^{d}$ be the $d$-dimensional periodic square lattice of size $N$ and consider a system that consists of particles of two distinct types. The configuration space is $\mathcal{X}_{N}^{2}=\mathcal{X}_{N} \times \mathcal{X}_{N}$, where $\mathcal{X}_{N}=\{0,1\}^{\mathbb{T}_{N}^{d}}$. Its elements are denoted by $\tilde{\sigma} \equiv\left(\sigma_{1}, \sigma_{2}\right)=\left(\left\{\sigma_{1, x}\right\}_{x \in \mathbb{T}_{N}^{d}},\left\{\sigma_{2, x}\right\}_{x \in \mathbb{T}_{N}^{d}}\right)$. The generator of the Kawasaki dynamics of particles of a single type is defined by

$$
\left(L_{0}^{1} f\right)(\sigma)=\frac{1}{2} \sum_{x, y \in \mathbb{T}_{N}^{d}:|x-y|=1}\left\{f\left(\sigma^{x, y}\right)-f(\sigma)\right\}, \quad \sigma=\left\{\sigma_{x}\right\}_{x \in \mathbb{T}_{N}^{d}} \in \mathcal{X}_{N},
$$

for functions $f: \mathcal{X}_{N} \rightarrow \mathbb{R}$, and where $\sigma^{x, y} \in \mathcal{X}_{N}$ is defined from $\sigma \in \mathcal{X}_{N}$ as

$$
\left(\sigma^{x, y}\right)_{z}= \begin{cases}\sigma_{y} & \text { if } z=x \\ \sigma_{x} & \text { if } z=y \\ \sigma_{z} & \text { otherwise }\end{cases}
$$

The generator of the two-component system is given by $L_{N}=N^{2} L_{0}+K L_{G}$ with $K=K(N)$, where

$$
\begin{aligned}
& \left(L_{0} f\right)\left(\sigma_{1}, \sigma_{2}\right)=d_{1}\left(L_{0}^{1} f\left(\cdot, \sigma_{2}\right)\right)\left(\sigma_{1}\right)+d_{2}\left(L_{0}^{1} f\left(\sigma_{1}, \cdot\right)\right)\left(\sigma_{2}\right), \\
& \left(L_{G} f\right)\left(\sigma_{1}, \sigma_{2}\right)=\sum_{x \in \mathbb{T}_{N}^{d}} \sigma_{1, x} \sigma_{2, x}\left\{f\left(\sigma_{1}^{x}, \sigma_{2}^{x}\right)-f\left(\sigma_{1}, \sigma_{2}\right)\right\},
\end{aligned}
$$


for functions $f: \mathcal{X}_{N}^{2} \rightarrow \mathbb{R}$, and $d_{1}, d_{2}>0$. Here $\sigma^{x} \in \mathcal{X}_{N}$ is defined from $\sigma \in \mathcal{X}_{N}$ as

$$
\sigma_{z}^{x}= \begin{cases}1-\sigma_{x} & \text { if } z=x \\ \sigma_{z} & \text { if } z \neq x\end{cases}
$$

Let $\tilde{\sigma}^{N}(t)=\left(\sigma_{1}^{N}(t), \sigma_{2}^{N}(t)\right) \equiv\left(\sigma_{1, x}^{N}(t), \sigma_{2, x}^{N}(t)\right)_{x \in \mathbb{T}_{N}^{d}}$ be the Markov process on $\mathcal{X}_{N}^{2}$ generated by $L_{N}$. The macroscopic empirical measures on $\mathbb{T}^{d}:=(\mathbb{R} / \mathbb{Z})^{d} \equiv[0,1)^{d}$ associated with a configuration $\tilde{\sigma}=\left(\sigma_{1}, \sigma_{2}\right) \in \mathcal{X}_{N}^{2}$ are defined by

$$
\alpha_{i}^{N}(d r ; \tilde{\sigma})=\frac{1}{N^{d}} \sum_{x \in \mathbb{T}_{N}^{d}} \sigma_{i, x} \delta_{\frac{x}{N}}(d r), \quad r \in \mathbb{T}^{d}, i=1,2 .
$$

The goal is to study the limit of the macroscopic empirical measures of the process $\tilde{\sigma}^{N}(t)$ as $N \rightarrow \infty$, with properly scaled $K(N)$.

1.2. Main result. We first summarize our assumptions on the initial distribution of $\tilde{\sigma}^{N}(0)$.

(A1) Let $u_{i}^{N}(0, \cdot)=\left\{u_{i}^{N}(0, x)\right\}_{x \in \mathbb{T}_{N}^{d}}, i=1,2$ be given and satisfy two bounds

$$
e^{-c_{1} K} \leq u_{i}^{N}(0, x) \leq c_{2} \quad \text { and } \quad\left|\nabla^{N} u_{i}^{N}(0, x)\right| \leq C_{0} K
$$

for every $i=1,2, x \in \mathbb{T}_{N}^{d}$ with $c_{1}>0,0<c_{2}<1, C_{0}>0$, where $K=K(N)$ satisfies $(\mathrm{A} 4)_{\delta}$ below $(\delta>0), \nabla^{N}$ is defined as

$$
\nabla^{N} u(x)=\left\{N\left(u\left(x+e_{i}\right)-u(x)\right)\right\}_{i=1}^{d}
$$

and $e_{i} \in \mathbb{Z}^{d}$ are the unit vectors in the $i$ th positive direction.

(A2) Let $\mu_{0}^{N}$ be the distribution of $\tilde{\sigma}^{N}(0)$ on $\mathcal{X}_{N}^{2}$ and let $\nu_{0}^{N}=\nu_{u_{1}^{N}(0, \cdot), u_{2}^{N}(0, \cdot)}$ be the Bernoulli measure on $\mathcal{X}_{N}^{2}$ with means $u_{1}^{N}(0, \cdot), u_{2}^{N}(0, \cdot)$ given as above. We assume the relative entropy defined in (2.1) satisfies $H\left(\mu_{0}^{N} \mid \nu_{0}^{N}\right)=$ $O\left(N^{d-\delta_{0}}\right)$ as $N \rightarrow \infty$ with some $\delta_{0}>0$.

(A3) We assume $u_{i}^{N}(0, r), r \in \mathbb{T}^{d}$ defined from $u_{i}^{N}(0, x)$ through (4.1) converge to some $u_{i}(0, r)$ weakly in $L^{2}\left(\mathbb{T}^{d}\right)$ as $N \rightarrow \infty$, for $i=1,2$, respectively.

$(\mathrm{A} 4)_{\delta} K=K(N)$ satisfies $1 \leq K(N) \leq \delta(\log N)^{1 / 2}$ and $K(N) \rightarrow \infty$ as $N \rightarrow \infty$.

Our main theorem is formulated as follows.

Theorem 1.1. We assume the four conditions (A1)-(A3),(A4) $\delta$ with $\delta>0$ chosen sufficiently small depending on $T>0$. Then, we have the following.

(1) The macroscopic empirical measures $\alpha_{i}^{N}(t, d r):=\alpha_{i}^{N}\left(d r ; \tilde{\sigma}^{N}(t)\right)$ of the process $\tilde{\sigma}^{N}(t)$ converge to $u_{i}(t, r) d r$, respectively, for $i=1,2$, that is

$$
\lim _{N \rightarrow \infty} P\left(\left|\left\langle\alpha_{i}^{N}(t), \varphi\right\rangle-\left\langle u_{i}(t), \varphi\right\rangle\right|>\varepsilon\right)=0, \quad i=1,2,
$$

for every $\varepsilon>0, t \in[0, T]$ and $\varphi \in C^{\infty}\left(\mathbb{T}^{d}\right)$, and $u_{1}(t, r) u_{2}(t, r)=0$ a.e. $r$ holds, where $\langle\alpha, \varphi\rangle$ and $\langle u, \varphi\rangle$ stand for the integrals over $\mathbb{T}^{d}$.

(2) $w(t, r):=u_{1}(t, r)-u_{2}(t, r)$ is the unique weak solution of

$$
\left\{\begin{array}{l}
\partial_{t} w=\Delta \mathcal{D}(w), \quad \text { in } \mathbb{T}^{d} \\
w(0, r)=u_{1}(0, r)-u_{2}(0, r)
\end{array}\right.
$$

where $\Delta$ is the Laplacian on $\mathbb{T}^{d}$, and $\mathcal{D}(s)=d_{1} s, s \geq 0$ and $=d_{2} s, s<0$.

The weak solution of (1.3) is defined as follows. 
Definition 1.2. We call $w=w(t, r)$ a weak solution of (1.3) if it satisfies

(i) $w \in L^{\infty}\left([0, T] \times \mathbb{T}^{d}\right)$ for every $T>0$;

(ii) For all $T>0$ and $\psi \in C^{1,2}\left([0, T] \times \mathbb{T}^{d}\right)$ such that $\psi(T, r)=0$ for all $r$, we have

$$
\int_{0}^{T} \int_{\mathbb{T}^{d}}\left(w \partial_{t} \psi+\mathcal{D}(w) \Delta \psi\right) d r d t=-\int_{\mathbb{T}^{d}} w_{0} \psi(0, r) d r .
$$

The uniqueness of the weak solution of (1.3) is shown in Crooks et al. (2004), Corollary 3.8. As pointed out in Dancer et al. (1999), (1.3) is the weak formulation of the following two-phase Stefan problem for $u_{1}$ and $u_{2}$ :

$$
\left\{\begin{array}{l}
\partial_{t} u_{1}=d_{1} \Delta u_{1}, \quad \text { on } D_{1}(t)=\left\{r \in \mathbb{T}^{d} ; u_{1}(t, r)>0, u_{2}(t, r)=0\right\}, \\
\partial_{t} u_{2}=d_{2} \Delta u_{2}, \quad \text { on } D_{2}(t)=\left\{r \in \mathbb{T}^{d} ; u_{1}(t, r)=0, u_{2}(t, r)>0\right\}, \\
u_{1}=u_{2}=0, \quad \text { on } \Gamma(t):=\partial D_{1}(t)=\partial D_{2}(t), \\
d_{1} \partial_{n} u_{1}=-d_{2} \partial_{n} u_{2}, \quad \text { on } \Gamma(t),
\end{array}\right.
$$

where $n$ is the unit normal vector on $\Gamma(t)$ directed to $D_{1}(t)$. Indeed, if the system (1.4) has a smooth solution, that is, if $\Gamma(t)$ is smooth, $u_{i}(t, r), i=1,2$, are smooth in $D_{i}(t)$ and continuous on $\mathbb{T}^{d}$, then it determines a weak solution.

The proof of Theorem 1.1 is divided into two parts as we mentioned above. The main task is to show that the relative entropy of our system compared with the local equilibria defined through the discretized hydrodynamic equation (2.3) behaves as $o\left(N^{d}\right)$, namely, the relative entropy per volume tends to 0 as $N \rightarrow \infty$. This is formulated in Theorem 2.2 and shown in Sections 2 and 3. Once this is shown, one can prove that the macroscopic empirical measures $\alpha_{i}^{N}(t)$ is close to the solution of (2.3), see Section 4. In the last Section 5, we show that the solution of (2.3) converges to the weak solution of (1.3).

A related model with instantaneous annihilation was studied by Funaki (1999) and the same equation (1.3) was derived in the limit. Briefly saying, $1 \ll K \ll N$ in our model, while $1 \ll N \ll K=\infty$ in Funaki (1999). Sasada (2010) considered the model with non-instantaneous annihilation together with creation of two distinct types of particles.

\section{Relative entropy method}

The relative entropy of two probability measures $\mu$ and $\nu$ on $\mathcal{X}_{N}^{2}$ is defined as

$$
H(\mu \mid \nu):=\int_{\mathcal{X}_{N}^{2}} \frac{d \mu}{d \nu} \log \frac{d \mu}{d \nu} \cdot d \nu
$$

For a probability measure $\nu$ on $\mathcal{X}_{N}^{2}$, the Dirichlet form $\mathcal{D}(f ; \nu), f: \mathcal{X}_{N}^{2} \rightarrow \mathbb{R}$, associated to the generator $L_{0}$ is defined as

$$
\begin{aligned}
& \mathcal{D}(f ; \nu) \\
& =\frac{1}{4} \sum_{\substack{x, y \in \mathbb{T}_{N}^{d} \\
|x-y|=1}} \int_{\mathcal{X}_{N}^{2}}\left[d_{1}\left\{f\left(\sigma_{1}^{x, y}, \sigma_{2}\right)-f\left(\sigma_{1}, \sigma_{2}\right)\right\}^{2}+d_{2}\left\{f\left(\sigma_{1}, \sigma_{2}^{x, y}\right)-f\left(\sigma_{1}, \sigma_{2}\right)\right\}^{2}\right] d \nu .
\end{aligned}
$$

Let $\mu_{t}=\mu_{t}^{N}$ be the law of $\left(\sigma_{1}^{N}(t), \sigma_{2}^{N}(t)\right)$ generated by $L_{N}=N^{2} L_{0}+K L_{G}$ on $\mathcal{X}_{N}^{2}$.

We have the following estimate on the time derivative of the relative entropy. See Funaki (2018), Funaki and Tsunoda (2018) for the proof. 
Proposition 2.1. For any probability measures $\left\{\nu_{t}\right\}$ and $m$ on $\mathcal{X}_{N}^{2}$ both with full supports in $\mathcal{X}_{N}^{2}$, we have

$$
\frac{d}{d t} H\left(\mu_{t} \mid \nu_{t}\right) \leq-2 N^{2} \mathcal{D}\left(\sqrt{\frac{d \mu_{t}}{d \nu_{t}}} ; \nu_{t}\right)+\int_{\mathcal{X}_{N}^{2}}\left(L_{N}^{*, \nu_{t}} 1-\partial_{t} \log \psi_{t}\right) d \mu_{t} .
$$

where $L_{N}^{*, \nu_{t}}$ is the adjoint of $L_{N}$ on $L^{2}\left(\nu_{t}\right)$ and $\psi_{t}:=\frac{d \nu_{t}}{d m}$.

This estimate was first used by Guo, Papanicolaou and Varadhan taking $\nu_{t}$ to be a global equilibrium which is independent of $t$ and then by Yau dropping the negative Dirichlet form term, see Funaki (2018). Then Jara and Menezes introduced (2.2) as a combination of these two estimates, cf. Jara and Menezes (2018).

We use (2.2) with the following Bernoulli measures $\nu_{t}$. Let $u_{i}^{N}(t)=\left\{u_{i}(t, x)=\right.$ $\left.u_{i}^{N}(t, x)\right\}_{x \in \mathbb{T}_{N}^{d}}, i=1,2$ be the solution of the system of the discretized hydrodynamic equation:

$$
\partial_{t} u_{i}^{N}(t, x)=d_{i} \Delta^{N} u_{i}^{N}(t, x)-K u_{1}^{N}(t, x) u_{2}^{N}(t, x), \quad i=1,2
$$

where $\Delta^{N}=N^{2} \Delta$ and

$$
(\Delta u)(x)=\sum_{y \in \mathbb{T}_{N}^{d}:|y-x|=1}(u(y)-u(x)) .
$$

Note that (2.3) is a discrete version of (1.1). We define $\nu_{t}=\nu_{u_{1}(t, \cdot), u_{2}(t, \cdot)}$, where we denote by $\nu_{u_{1}(\cdot), u_{2}(\cdot)}$ for $u_{i}(\cdot)=\left\{u_{i}(x)\right\}_{x \in \mathbb{T}_{N}^{d}}, i=1,2$ the Bernoulli measure on $\mathcal{X}_{N}^{2}$ such that

$$
\nu_{u_{1}(\cdot), u_{2}(\cdot)}\left(\sigma_{i, x}=1\right)=u_{i}(x),
$$

for every $x \in \mathbb{T}_{N}^{d}$ and $i=1,2$.

The main result in the probabilistic part is the following Theorem.

Theorem 2.2. Assume the initial distribution verifies the Hypothesis of Theorem 1.1. Then, we have

$$
H\left(\mu_{t}^{N} \mid \nu_{t}^{N}\right)=o\left(N^{d}\right), \quad t \in[0, T]
$$

as $N \rightarrow \infty$.

The proof of this theorem needs some preliminary results proved in the following subsections.

2.1. Calculation of the second term in (2.2). We define the normalized variables $\omega_{i, x, t}$ by

$$
\omega_{i, x, t}=\frac{\bar{\sigma}_{i, x}(t)}{\chi\left(u_{i}(t, x)\right)}, \quad \bar{\sigma}_{i, x}(t)=\sigma_{i, x}(t)-u_{i}(x, t), \quad i=1,2,
$$

where $\chi(u)=u(1-u)$ for $u \in(0,1)$.

In this subsection we prove the following proposition.

\section{Proposition 2.3.}

$$
L_{N}^{*, \nu_{t}} 1-\partial_{t} \log \psi_{t}=V_{1}(t)+V_{2}(t)+V(t)
$$


with

$$
\begin{aligned}
& V_{i}(t)=-\frac{d_{i} N^{2}}{2} \sum_{x, y \in \mathbb{T}_{N}^{d}:|x-y|=1}\left(u_{i}(t, y)-u_{i}(t, x)\right)^{2} \omega_{i, x, t} \omega_{i, y, t}, \quad i=1,2 \\
& V(t)=K \sum_{x \in \mathbb{T}_{N}^{d}}\left(u_{1}(t, x)+u_{2}(t, x)-1\right) u_{1}(t, x) u_{2}(t, x) \omega_{1, x, t} \omega_{2, x, t} .
\end{aligned}
$$

Proof: We first compute $L_{N}^{*, \nu_{t}} g$ for a generic function $g$ on $\mathcal{X}_{N}^{2}$, calling $\nu=$ $\nu_{u_{1}(\cdot), u_{2}(\cdot)}$.

For $L_{G}$ we have

$$
\int g L_{G} f d \nu=\sum_{\sigma_{1}, \sigma_{2}} g\left(\sigma_{1}, \sigma_{2}\right) \sum_{x \in \mathbb{T}_{N}^{d}} \sigma_{1, x} \sigma_{2, x}\left\{f\left(\sigma_{1}^{x}, \sigma_{2}^{x}\right)-f\left(\sigma_{1}, \sigma_{2}\right)\right\} \nu\left(\sigma_{1}, \sigma_{2}\right) .
$$

By making the change of variables $\eta_{1}=\sigma_{1}^{x}$ and $\eta_{2}=\sigma_{2}^{x}$, the sum containing $f\left(\sigma_{1}^{x}, \sigma_{2}^{x}\right)$ can be rewritten as

$$
\sum_{\eta_{1}, \eta_{2}} g\left(\eta_{1}^{x}, \eta_{2}^{x}\right) \sum_{x \in \mathbb{T}_{N}^{d}}\left(1-\eta_{1, x}\right)\left(1-\eta_{2, x}\right) f\left(\eta_{1}, \eta_{2}\right) \nu\left(\eta_{1}^{x}, \eta_{2}^{x}\right)
$$

Next observe that for $\eta_{1}, \eta_{2}$ satisfying $\eta_{1, x}=\eta_{2, x}=0$,

$$
\nu\left(\eta_{1}^{x}, \eta_{2}^{x}\right)=\frac{u_{1}(x) u_{2}(x)}{\left(1-u_{1}(x)\right)\left(1-u_{2}(x)\right)} \nu\left(\eta_{1}, \eta_{2}\right)
$$

Thus

$$
\begin{array}{r}
L_{G}^{*, \nu} g\left(\sigma_{1}, \sigma_{2}\right)=\sum_{x \in \mathbb{T}_{N}^{d}}\left\{\frac{u_{1}(x) u_{2}(x)}{\left(1-u_{1}(x)\right)\left(1-u_{2}(x)\right)}\left(1-\sigma_{1, x}\right)\left(1-\sigma_{2, x}\right) g\left(\sigma_{1}^{x}, \sigma_{2}^{x}\right)\right. \\
\left.-\sigma_{1, x} \sigma_{2, x} g\left(\sigma_{1}, \sigma_{2}\right)\right\} .
\end{array}
$$

Using the above equality with $g \equiv 1$ and writing $\bar{\sigma}_{i, x}=\omega_{i, x, t} \chi\left(u_{i}(\cdot)\right)$ (recall (2.4)) we get

$$
\begin{aligned}
K L_{G}^{*, \nu_{u_{1}(\cdot), u_{2}(\cdot)}} 1= & -K \sum_{x \in \mathbb{T}_{N}^{d}} u_{1}(x) u_{2}(x)\left(\omega_{1, x}+\omega_{2, x}\right) \\
& +K \sum_{x \in \mathbb{T}_{N}^{d}}\left(u_{1}(x)+u_{2}(x)-1\right) u_{1}(x) u_{2}(x) \omega_{1, x} \omega_{2, x} .
\end{aligned}
$$

For the Kawasaki part, from the computation in Funaki (2018) or Funaki and Tsunoda (2018), we obtain

$$
\begin{aligned}
L_{0}^{*, \nu} 1= & -\frac{d_{1}}{2} \sum_{\substack{x, y \in \mathbb{T}_{N}^{d} \\
|x-y|=1}}\left(u_{1}(y)-u_{1}(x)\right)^{2} \omega_{1, x} \omega_{1, y}+d_{1} \sum_{x \in \mathbb{T}_{N}^{d}}\left(\Delta u_{1}\right)(x) \omega_{1, x} \\
& -\frac{d_{2}}{2} \sum_{\substack{x, y \in \mathbb{T}_{N}^{d} \\
|x-y|=1}}\left(u_{2}(y)-u_{2}(x)\right)^{2} \omega_{2, x} \omega_{2, y}+d_{2} \sum_{x \in \mathbb{T}_{N}^{d}}\left(\Delta u_{2}\right)(x) \omega_{2, x} .
\end{aligned}
$$


We next observe that

$$
\partial_{t} \log \psi_{t}(\eta)=\sum_{x \in \mathbb{T}_{N}^{d}} \partial_{t} u_{1}(t, x) \omega_{1, x, t}+\sum_{x \in \mathbb{T}_{N}^{d}} \partial_{t} u_{2}(t, x) \omega_{2, x, t}
$$

This equality is proved similarly to Funaki (2018) or Funaki and Tsunoda (2018). By using (2.3) the linear terms in $\omega$ cancel and we finally obtain (2.5).

2.2. Estimates on the solution of (2.3). Let $\left(u_{1}(t), u_{2}(t)\right)=\left(u_{1}^{N}(t, x), u_{2}^{N}(t, x)\right)$ be the solution of the discretized hydrodynamic equation (2.3). We derive estimates on $\left(u_{1}(t), u_{2}(t)\right)$ and their gradients. First two lemmas, especially taking $c=c_{2}<1$ with $c_{2}$ in (A1), are useful to estimate $1 / \chi\left(u_{i}^{N}(t, x)\right)$ appearing in the definition of $\omega_{i, x, t}$ from above.

Lemma 2.4. If the initial values satisfy $0 \leq u_{i}^{N}(0, x) \leq c$ for every $x \in \mathbb{T}_{N}^{d}$ and $i=1,2$ with some $c>0$, we have

$$
0 \leq u_{i}^{N}(t, x) \leq c,
$$

for every $t \geq 0, x \in \mathbb{T}_{N}^{d}$ and $i=1,2$.

Proof: One can apply the maximum principle in our discrete setting, cf. Crooks et al. (2004), Lemma 2.1. Also, a similar argument to the proof of the next lemma works.

Lemma 2.5. If the initial values satisfy $0<u_{0} \leq u_{i}^{N}(0, x) \leq 1$ for every $x \in \mathbb{T}_{N}^{d}$ and $i=1,2$, we have

$$
u_{i}^{N}(t, x) \geq \underline{u}(t):=u_{0} e^{-K t},
$$

for every $t \geq 0, x \in \mathbb{T}_{N}^{d}$ and $i=1,2$.

Proof: From (2.3) and $u_{2}(t, x) \leq 1$, since $\underline{\mathrm{u}}(t)$ satisfies $\partial_{t} \underline{\mathrm{u}}(t)=-K \underline{\mathrm{u}}(t)$, we have

$$
\begin{aligned}
\partial_{t}\left(u_{1}(t, x)-\underline{\mathrm{u}}(t)\right) & =d_{1} \Delta^{N}\left(u_{1}(t, x)-\underline{\mathrm{u}}(t)\right)-K\left(u_{1}(t, x) u_{2}(t, x)-\underline{\mathrm{u}}(t)\right) \\
& \geq d_{1} \Delta^{N}\left(u_{1}(t, x)-\underline{\mathrm{u}}(t)\right)-K\left(u_{1}(t, x)-\underline{\mathrm{u}}(t)\right) .
\end{aligned}
$$

Assume that $u_{1}(s, y)>\underline{\mathrm{u}}(s)$ holds for $0 \leq s<t$ and every $y \in \mathbb{T}_{N}^{d}$, and at some $x$ and $t, u_{1}(t, x)=\underline{\mathrm{u}}(t)$ holds. Then, $\Delta^{N}\left(u_{1}(t, x)-\underline{\mathrm{u}}(t)\right) \geq 0$ and $-K\left(u_{1}(t, x)-\right.$ $\underline{\mathrm{u}}(t))=0$. Therefore, $\partial_{t}\left(u_{1}(t, x)-\underline{\mathrm{u}}(t)\right) \geq 0$. This means that $u_{1}(t, x)-\underline{\mathrm{u}}(t)$ is increasing and $u_{1}(t, x)$ can not be below $\underline{\mathrm{u}}(t)$. Same argument works for $u_{2}(t, x)$.

Let $p^{N}(t, x, y)$ be the discrete heat kernel corresponding to $\Delta^{N}$ on $\mathbb{T}_{N}^{d}$. Then, we have the following estimate, which is global in $t$.

Lemma 2.6. There exist $C, c>0$ such that

$$
\left|\nabla^{N} p^{N}(t, x, y)\right| \leq \frac{C}{\sqrt{t}} p^{N}(c t, x, y), \quad t>0,
$$

where $\nabla^{N}$ is defined by (1.2).

Proof: Let $p(t, x, y)$ be the heat kernel corresponding to the discrete Laplacian $\Delta$ on $\mathbb{Z}^{d}$. Then, we have the estimate

$$
|\nabla p(t, x, y)| \leq \frac{C}{\sqrt{1 \vee t}} p(c t, x, y), \quad t>0, x, y \in \mathbb{Z}^{d},
$$

with some constants $C, c>0$, independent of $t$ and $x, y$, where $\nabla=\nabla^{1}$. This should be well-known, but we refer to Delmotte and Deuschel (2005) Theorem 1.1 
(1.4) which discusses general case with random coefficients, see also Stroock and Zheng (1997). Then, since

$$
p^{N}(t, x, y)=\sum_{k \in(N \mathbb{Z})^{d}} p\left(N^{2} t, x, y+k\right),
$$

the result follows.

We have the following estimate, though it might not be the best possible one.

Proposition 2.7. The gradients of the solution of (2.3) are estimated as

$$
\left|\nabla^{N} u_{i}^{N}(t, x)\right| \leq K\left(C_{0}+C \sqrt{t}\right), \quad t>0, i=1,2,
$$

if $\left|\nabla^{N} u_{i}^{N}(0, x)\right| \leq C_{0} K$ holds.

Proof: From Duhamel's formula, we have

$$
\begin{aligned}
u_{i}^{N}(t, x)= & \sum_{y \in \mathbb{T}_{N}^{d}} u_{i}^{N}(0, y) p^{N}\left(d_{i} t, x, y\right) \\
& -K \int_{0}^{t} d s \sum_{y \in \mathbb{T}_{N}^{d}} u_{1}^{N}(s, y) u_{2}^{N}(s, y) p^{N}\left(d_{i}(t-s), x, y\right) .
\end{aligned}
$$

By noting the symmetry of $p^{N}$ in $(x, y)$ and $0 \leq u_{1}^{N}(s, x), u_{2}^{N}(s, x) \leq 1$, this shows

$$
\begin{aligned}
\left|\nabla^{N} u_{i}^{N}(t, x)\right| \leq & \sum_{y \in \mathbb{T}_{N}^{d}}\left|\nabla^{N} u_{i}^{N}(0, y)\right| p^{N}\left(d_{i} t, x, y\right) \\
& +K \int_{0}^{t} d s \sum_{y \in \mathbb{T}_{N}^{d}}\left|\nabla^{N} p^{N}\left(d_{i}(t-s), x, y\right)\right| .
\end{aligned}
$$

Thus, from Lemma 2.6, we obtain the desired estimate.

2.3. Proof of Theorem 2.2. Notation: We simply denote $\mu_{t}=\mu_{t}^{N}, \nu_{t}=\nu_{t}^{N}$ and set $f_{t} \equiv f_{t}^{N}:=\frac{d \mu_{t}^{N}}{d \nu_{t}^{N}}$.

Recalling Proposition 2.3, and using the estimates of subsection 2.2, in Section 3 we prove the following Theorem.

Theorem 2.8. For $\alpha$ and $\kappa>0$ small, if $d \geq 2$, there is $C_{\alpha, \kappa}>0$ so that

$$
\int_{\mathcal{X}_{N}^{2}} V(t) d \mu_{t} \leq \alpha N^{2} \mathcal{D}\left(\sqrt{f_{t}} ; \nu_{t}\right)+C_{\alpha, \kappa} K H\left(\mu_{t} \mid \nu_{t}\right)+N^{d-1+\kappa},
$$

and also

$$
\int_{\mathcal{X}_{N}^{2}}\left[V_{1}(t)+V_{2}(t)\right] d \mu_{t} \leq \alpha N^{2} \mathcal{D}\left(\sqrt{f_{t}} ; \nu_{t}\right)+C_{\alpha, \kappa} K^{2} H\left(\mu_{t} \mid \nu_{t}\right)+N^{d-1+\kappa} .
$$

The term $N^{d-1+\kappa}$ is replaced by $N^{\frac{1}{2}+\kappa}$ when $d=1$.

By using Proposition 2.1, (2.5) and the above Theorem, we obtain

$$
\frac{d}{d t} H\left(\mu_{t} \mid \nu_{t}\right) \leq C K^{2} H\left(\mu_{t} \mid \nu_{t}\right)+O\left(N^{d-\delta_{1}}\right),
$$


with $0<\delta_{1}<1$. We have chosen $\alpha \in(0,1)$ so that the terms of Dirichlet forms with a positive sign are absorbed by the one with negative sign in (2.2). Thus, Gronwall's inequality shows

$$
H\left(\mu_{t} \mid \nu_{t}\right) \leq\left(H\left(\mu_{0} \mid \nu_{0}\right)+t O\left(N^{d-\delta_{1}}\right)\right) e^{C K^{2} t} .
$$

Noting $e^{C K^{2} t} \leq N^{C t \delta^{2}}$ from $1 \leq K=K(N) \leq \delta(\log N)^{1 / 2}$ and $H\left(\mu_{0} \mid \nu_{0}\right)=$ $O\left(N^{d-\delta_{0}}\right)$ by the assumption, this concludes the proof of Theorem 2.2, if $\delta=\delta_{T}>0$ is small enough such that $C T \delta^{2}<\delta_{0} \wedge \delta_{1}$.

\section{Proof of Theorem 2.8}

We split the proof in two subsections.

3.1. Proof of (2.8). We omit the dependence on $t$ and define

$$
V:=K \sum_{x \in \mathbb{T}_{N}^{d}} \tilde{\omega}_{1, x} \omega_{2, x}
$$

where $\tilde{\omega}_{1, x}=\left(u_{1}(x)+u_{2}(x)-1\right) u_{1}(x) u_{2}(x) \omega_{1, x}$.

The first step is to replace $V$ by its local sample average $V^{\ell}$ defined by

$$
V^{\ell}:=K \sum_{x \in \mathbb{T}_{N}^{d}}{\overleftarrow{\left(\tilde{\omega}_{1}\right)_{x, \ell}}}_{\left(_{\left(\omega_{2}\right.}\right)_{x, \ell}}
$$

where

$$
\vec{g}_{x, \ell}:=\frac{1}{\left|\Lambda_{\ell}\right|} \sum_{y \in \Lambda_{\ell}} g_{x+y}, \quad \overleftarrow{g}_{x, \ell}:=\frac{1}{\left|\Lambda_{\ell}\right|} \sum_{y \in \Lambda_{\ell}} g_{x-y}
$$

for a function $g=\left\{g_{x}\left(\sigma_{1}, \sigma_{2}\right)\right\}$ and $\Lambda_{\ell}=[0, \ell-1]^{d} \cap \mathbb{Z}^{d}$.

Proposition 3.1. We assume the conditions of Theorem 2.2, in particular, we take $\delta>0$ sufficiently small. Let $\nu=\nu_{u_{1}(\cdot), u_{2}(\cdot)}, d \mu=f d \nu$ (recall we omit $t$ ) and we choose $\ell=N^{\frac{1}{d}-\kappa^{\prime}}$ with $\kappa^{\prime}(=\kappa / d)>0$ when $d \geq 2$ and $\ell=N^{\frac{1}{2}-\kappa}$ when $d=1$, with small $\kappa>0$. Then the cost of the replacement is estimated as

$$
\int\left(V-V^{\ell}\right) f d \nu \leq \alpha N^{2} \mathcal{D}(\sqrt{f} ; \nu)+C_{\alpha, \kappa}\left(H(\mu \mid \nu)+N^{d-1+\kappa}\right),
$$

for every $\alpha, \kappa>0$ with some $C_{\alpha, \kappa}>0$ when $d \geq 2$ and the last $N^{d-1+\kappa}$ is replaced by $N^{\frac{1}{2}+\kappa}$ when $d=1$.

The first tool to show this proposition is the flow lemma for the telescopic sum. We call $\Phi=\{\Phi(x, y)\}_{b=\{x, y\} \in G^{*}}$ a flow on a finite set $G$ connecting two probability measures $p$ and $q$ on $G$ if $\Phi(x, y)=-\Phi(y, x)$ hold for all $\{x, y\} \in G^{*}$ and $\sum_{z \in G} \Phi(x, z)=p(x)-q(x)$ hold for all $x \in G$, where $G^{*}$ is the set of all bonds in $G$. The following lemma is found in Appendix G of Jara and Menezes (2018), see also Funaki (2018), Funaki and Tsunoda (2018).

Lemma 3.2. (Flow lemma) There exists a flow $\Phi^{\ell}$ on $\Lambda_{2 \ell}:=\{0,1, \ldots, 2 \ell-1\}^{d}$ connecting $\delta_{0}$ and $q_{\ell}:=p_{\ell} * p_{\ell}, p_{\ell}(x)=\frac{1}{\left|\Lambda_{\ell}\right|} 1_{\Lambda_{\ell}}(x)$, such that

$$
\sum_{x \in \Lambda_{2 \ell-1}} \sum_{j=1}^{d} \Phi^{\ell}\left(x, x+e_{j}\right)^{2} \leq C_{d} g_{d}(\ell),
$$


where $e_{j}$ is a unit vector to $j$ th positive direction, and $g_{d}(\ell)=\ell$ when $d=1, \log \ell$ when $d=2$ and 1 when $d \geq 3$.

Remark 3.3. (1) When $d=1$, the flow $\Phi^{\ell}$ on $\Lambda_{\ell+1}=\{0,1, \ldots, \ell\}$ connecting $\delta_{0}$ and $p_{\ell}(x)=\frac{1}{\ell} 1_{\{1, \ldots, \ell\}}(x)$ is given by $\Phi^{\ell}(x, x+1)=\frac{\ell-x}{\ell}, 0 \leq x \leq \ell-1$. Indeed, the condition on $\Phi^{\ell}$ is

$$
\Phi^{\ell}(x, x+1)+\Phi^{\ell}(x, x-1)=\delta_{0}(x)-p_{\ell}(x), \quad x \in \Lambda_{\ell},
$$

with $\Phi^{\ell}(\ell, \ell+1)=\Phi^{\ell}(0,-1)=0$. Or equivalently, recalling that $\Phi^{\ell}(x, x-1)=$ $-\Phi^{\ell}(x-1, x)$ and setting $\tilde{\Phi}(x):=\Phi^{\ell}(x, x+1)$, the condition is

$$
\begin{aligned}
& \nabla \tilde{\Phi}(x)(=\tilde{\Phi}(x)-\tilde{\Phi}(x-1))=-\frac{1}{\ell}, \quad 1 \leq x \leq \ell, \\
& \tilde{\Phi}(0)=1, \tilde{\Phi}(\ell)=0
\end{aligned}
$$

i.e., the gradient of $\tilde{\Phi}$ is a constant so that $\tilde{\Phi}$ is an affine function. This equation is easily solved and we obtain $\tilde{\Phi}(x)=\frac{\ell-x}{\ell}$.

(2) In Lemma 3.2, we are concerned with $q_{\ell}$ instead of $p_{\ell}$. When $d=1$,

$$
\begin{aligned}
q_{\ell}(x) & =\sum_{y \in \mathbb{T}_{N}^{d}} p_{\ell}(x-y) p_{\ell}(y)=\frac{1}{\ell^{2}} \sum_{1 \leq x-y \leq \ell, 1 \leq y \leq \ell} 1 \\
& =\frac{1}{\ell^{2}} \sharp\{y: 1 \leq y \leq \ell, x-\ell \leq y \leq x-1\} \\
& = \begin{cases}\frac{x-1}{\ell^{2}} & (\text { if } x-\ell \leq 1, \text { i.e. } x \leq \ell+1), \\
\frac{2 \ell+1-x}{\ell^{2}} & \text { (if } x-\ell \geq 1, \text { i.e. } x \geq \ell+1),\end{cases}
\end{aligned}
$$

i.e., $q_{\ell}$ is piecewise affine. Therefore, its integration $\Phi^{\ell}$ is piecewise quadratic.

Note that

$$
\begin{aligned}
\left(g * p_{\ell}\right)(x) & =\sum_{y \in \mathbb{T}_{N}^{d}} g_{x-y} p_{\ell}(y) \\
& =\frac{1}{\left|\Lambda_{\ell}\right|} \sum_{y \in \Lambda_{\ell}} g_{x-y}=\overleftarrow{g}_{x, \ell}
\end{aligned}
$$

and similarly $\left(g * \hat{p}_{\ell}\right)(x)=\vec{g}_{x, \ell}$, where $\hat{p}_{\ell}(y):=p_{\ell}(-y)$. Therefore,

$$
\begin{aligned}
V^{\ell} & =K \sum_{x \in \mathbb{T}_{N}^{d}}{\overleftarrow{\left(\tilde{\omega}_{1}\right)_{x, \ell}}}{\overrightarrow{\left(\omega_{2}\right.}}_{x, \ell} \\
& =K \sum_{y \in \mathbb{T}_{N}^{d}} \tilde{\omega}_{1, y}\left(\omega_{2} * \hat{q}_{\ell}\right)(y)
\end{aligned}
$$


where $q_{\ell}$ is defined as in Lemma 3.2 and $\hat{q}_{\ell}(y):=q_{\ell}(-y)$. Accordingly, from Lemma 3.2 and $\Phi^{\ell}\left(y, y-e_{j}\right)=-\Phi^{\ell}\left(y-e_{j}, y\right)$, one can rewrite

$$
\begin{aligned}
V-V^{\ell} & =K \sum_{x \in \mathbb{T}_{N}^{d}} \tilde{\omega}_{1, x}\left\{\omega_{2, x}-\left(\omega_{2} * \hat{q}_{\ell}\right)(x)\right\} \\
& =K \sum_{x \in \mathbb{T}_{N}^{d}} \tilde{\omega}_{1, x}\left\{\omega_{2, x}-\sum_{y \in \mathbb{T}_{N}^{d}} \omega_{2, x+y} q_{\ell}(y)\right\} \\
& =K \sum_{x \in \mathbb{T}_{N}^{d}} \tilde{\omega}_{1, x} \sum_{y \in \mathbb{T}_{N}^{d}} \omega_{2, x+y}\left\{\delta_{0}(y)-q_{\ell}(y)\right\} \\
& =K \sum_{x \in \mathbb{T}_{N}^{d}} \tilde{\omega}_{1, x} \sum_{y \in \mathbb{T}_{N}^{d}} \omega_{2, x+y} \sum_{ \pm} \sum_{j=1}^{d} \Phi^{\ell}\left(y, y \pm e_{j}\right) \\
& =K \sum_{j=1}^{d} \sum_{x \in \mathbb{T}_{N}^{d}} \tilde{\omega}_{1, x} \sum_{y \in \mathbb{T}_{N}^{d}}\left(\omega_{2, x+y}-\omega_{2, x+y+e_{j}}\right) \Phi^{\ell}\left(y, y+e_{j}\right) \\
& =-K \sum_{j=1}^{d} \sum_{x \in \mathbb{T}_{N}^{d}}\left(\sum_{y \in \mathbb{T}_{N}^{d}} \tilde{\omega}_{1, x-y} \Phi^{\ell}\left(y, y+e_{j}\right)\right)\left\{\omega_{2, x+e_{j}}-\omega_{2, x}\right\} .
\end{aligned}
$$

Thus, we have shown

$$
V-V^{\ell}=-K \sum_{j=1}^{d} \sum_{x \in \mathbb{T}_{N}^{d}} h_{x}^{\ell, j}\left(\omega_{2, x+e_{j}}-\omega_{2, x}\right),
$$

where

$$
h_{x}^{\ell, j} \equiv h_{x}^{\ell, j}\left(\sigma_{1}\right)=\sum_{y \in \Lambda_{2 \ell-1}} \tilde{\omega}_{1, x-y} \Phi^{\ell}\left(y, y+e_{j}\right)
$$

We will use the property $h_{x}^{\ell, j}\left(\sigma_{2}^{x, x+e_{j}}\right)=h_{x}^{\ell, j}\left(\sigma_{2}\right)$, which is obvious since $h_{x}^{\ell, j}$ is a function of $\sigma_{1}$, see Lemma 3.5 below.

Another lemma we use is the integration by parts formula under the Bernoulli measure $\nu_{u_{1}(\cdot), u_{2}(\cdot)}$ on $\mathcal{X}_{N}^{2}$ with a spatially dependent mean. We will apply this formula for the function $h=h_{x}^{\ell, j}$. The formula is stated for general $h$ with an error caused by the non-constant property of $u_{2}(\cdot)$.

Lemma 3.4. (Integration by parts) Let $\nu=\nu_{u_{1}(\cdot), u_{2}(\cdot)}$ and assume $e^{-c_{1} K} \leq$ $u_{2}(x), u_{2}(y) \leq c_{2}$ holds for $x, y \in \mathbb{T}_{N}^{d}:|x-y|=1$ with some $c_{1}>0,0<c_{2}<1$. Then, for $h=h\left(\sigma_{1}, \sigma_{2}\right)$ and a probability density $f=f\left(\sigma_{1}, \sigma_{2}\right)$ with respect to $\nu$, we have

$$
\int h\left(\sigma_{2, y}-\sigma_{2, x}\right) f d \nu=\int h\left(\sigma_{1}, \sigma_{2}^{x, y}\right) \sigma_{2, x}\left(f\left(\sigma_{1}, \sigma_{2}^{x, y}\right)-f\left(\sigma_{1}, \sigma_{2}\right)\right) d \nu+R_{1},
$$

and the error term $R_{1}=R_{1, x, y}$ is bounded as

$$
\left|R_{1}\right| \leq C e^{2 c_{1} K}\left|\nabla_{x, y}^{1} u_{2}\right| \int\left|h\left(\sigma_{1}, \sigma_{2}\right)\right| f d \nu+\left\|h-h\left(\sigma_{1}, \sigma_{2}^{x, y}\right)\right\|_{\infty},
$$

with some $C=C_{c_{2}}>0$, where $\nabla_{x, y}^{1} u=u(x)-u(y)$. 
Proof: First we write

$$
\int h\left(\sigma_{2, y}-\sigma_{2, x}\right) f d \nu=\sum_{\sigma_{1}, \sigma_{2}} h\left(\sigma_{1}, \sigma_{2}\right)\left(\sigma_{2, y}-\sigma_{2, x}\right) f\left(\sigma_{1}, \sigma_{2}\right) \nu\left(\sigma_{1}, \sigma_{2}\right) .
$$

Then, by a change of variables $\zeta:=\sigma_{2}^{x, y}$ and writing $\zeta$ by $\sigma_{2}$ again, we have

$$
\sum_{\sigma_{2}} h\left(\sigma_{1}, \sigma_{2}\right) \sigma_{2, y} f\left(\sigma_{1}, \sigma_{2}\right) \nu_{2}\left(\sigma_{2}\right)=\sum_{\sigma_{2}} h\left(\sigma_{1}, \sigma_{2}^{x, y}\right) \sigma_{2, x} f\left(\sigma_{1}, \sigma_{2}^{x, y}\right) \nu_{2}\left(\sigma_{2}^{x, y}\right),
$$

where $\nu_{2}=\nu_{u_{2}(\cdot)}$ is a probability measure on $\mathcal{X}_{N}$, recall $\nu=\nu_{u_{1}(\cdot)} \otimes \nu_{u_{2}(\cdot)}$. To replace the last $\nu_{2}\left(\sigma_{2}^{x, y}\right)$ by $\nu_{2}\left(\sigma_{2}\right)$, we observe

$$
\begin{aligned}
& \frac{\nu_{2}\left(\sigma^{x, y}\right)}{\nu_{2}(\sigma)} \\
& =1_{\left\{\sigma_{x}=1, \sigma_{y}=0\right\}} \frac{\left(1-u_{2}(x)\right) u_{2}(y)}{u_{2}(x)\left(1-u_{2}(y)\right)}+1_{\left\{\sigma_{x}=0, \sigma_{y}=1\right\}} \frac{u_{2}(x)\left(1-u_{2}(y)\right)}{\left(1-u_{2}(x)\right) u_{2}(y)}+1_{\left\{\sigma_{x}=\sigma_{y}\right\}} \\
& =1+r_{x, y}(\sigma),
\end{aligned}
$$

with

$$
r_{x, y}(\sigma)=1_{\left\{\sigma_{x}=1, \sigma_{y}=0\right\}} \frac{u_{2}(y)-u_{2}(x)}{u_{2}(x)\left(1-u_{2}(y)\right)}+1_{\left\{\sigma_{x}=0, \sigma_{y}=1\right\}} \frac{u_{2}(x)-u_{2}(y)}{\left(1-u_{2}(x)\right) u_{2}(y)} .
$$

By the condition on $u_{2}$, this error is bounded as

$$
\left|r_{x y}(\sigma)\right| \leq C_{0} e^{c_{1} K}\left|\nabla_{x, y}^{1} u_{2}\right|, \quad C_{0}=C_{c_{2}}>0 .
$$

These computations are summarized as

$$
\begin{aligned}
& \int h\left(\sigma_{2, y}-\sigma_{2, x}\right) f d \nu=\int h\left(\sigma_{1}, \sigma_{2}^{x, y}\right) \sigma_{2, x} f\left(\sigma_{1}, \sigma_{2}^{x, y}\right)\left(1+r_{x y}\left(\sigma_{2}\right)\right) d \nu-\int h \sigma_{2, x} f d \nu \\
&= \int h\left(\sigma_{1}, \sigma_{2}^{x, y}\right) \sigma_{2, x}\left(f\left(\sigma_{1}, \sigma_{2}^{x, y}\right)-f\left(\sigma_{1}, \sigma_{2}\right)\right) d \nu \\
& \quad+\int\left(h\left(\sigma_{1}, \sigma_{2}^{x, y}\right)-h\left(\sigma_{1}, \sigma_{2}\right)\right) \sigma_{2, x} f d \nu+\int h\left(\sigma_{1}, \sigma_{2}^{x, y}\right) \sigma_{2, x} f\left(\sigma_{1}, \sigma_{2}^{x, y}\right) r_{x y}\left(\sigma_{2}\right) d \nu .
\end{aligned}
$$

The second term is bounded by $\left\|h\left(\sigma_{1}, \sigma_{2}^{x, y}\right)-h\left(\sigma_{1}, \sigma_{2}\right)\right\|_{\infty}$, since $\left|\sigma_{2, x}\right| \leq 1$ and $\int f d \nu=1$. For the third term denoted by $R_{0}$, applying the change of variables again, we have

$$
\begin{aligned}
\left|R_{0}\right| & =\left|\sum_{\sigma_{1}, \sigma_{2}} h\left(\sigma_{1}, \sigma_{2}\right) \sigma_{2, y} f\left(\sigma_{1}, \sigma_{2}\right) r_{x y}\left(\sigma_{2}^{x, y}\right) \nu\left(\sigma_{1}, \sigma_{2}^{x, y}\right)\right| \\
& =\left|\sum_{\sigma_{1}, \sigma_{2}} h\left(\sigma_{1}, \sigma_{2}\right) \sigma_{2, y} f\left(\sigma_{1}, \sigma_{2}\right) r_{x y}\left(\sigma_{2}^{x, y}\right)\left(1+r_{x y}\left(\sigma_{2}\right)\right) \nu\left(\sigma_{1}, \sigma_{2}\right)\right| \\
& \leq C_{0} e^{c_{1} K}\left|\nabla_{x, y}^{1} u_{2}\right|\left(1+C_{0} e^{c_{1} K}\left|\nabla_{x, y}^{1} u_{2}\right|\right) \int|h(\sigma)| f d \nu \\
& \leq C e^{2 c_{1} K}\left|\nabla_{x, y}^{1} u_{2}\right| \int|h(\sigma)| f d \nu,
\end{aligned}
$$

since $\left|\sigma_{2, y}\right| \leq 1$ and $\left|\nabla_{x, y}^{1} u_{2}\right| \leq 2 c_{2}$. This completes the proof.

We apply Lemma 3.4 to $V-V^{\ell}$ given in (3.3). Note that $h_{x}^{\ell, j}\left(\sigma_{1}\right)$ is invariant under the transform $\sigma_{2} \mapsto \sigma_{2}^{x, y}$. Since we have $\omega_{2, x}=\frac{\sigma_{2, x}-u_{2}(x)}{\chi\left(u_{2}(x)\right)}$ in (3.3) instead of 
$\sigma_{2, x}$ in Lemma 3.4, we need to estimate the error caused by the $x$-dependence of $\omega_{2, x}$ through $u_{2}(x)$.

Lemma 3.5. We assume that $\nu=\nu_{u_{1}(\cdot), u_{2}(\cdot)}$ satisfies the same condition as in Lemma 3.4. Then, we have

$$
\int h_{x}^{\ell, j}\left(\omega_{2, x+e_{j}}-\omega_{2, x}\right) f d \nu=\int h_{x}^{\ell, j} \frac{\sigma_{2, x}}{\chi\left(u_{2}(x)\right)}\left(f\left(\sigma_{1}, \sigma_{2}^{x, x+e_{j}}\right)-f\left(\sigma_{1}, \sigma_{2}\right)\right) d \nu+R_{2},
$$

and the error term $R_{2}=R_{2, x, j}$ is bounded as

$$
\left|R_{2}\right| \leq C e^{3 c_{1} K}\left|\nabla_{x, x+e_{j}}^{1} u_{2}\right| \int\left|h_{x}^{\ell, j}\left(\sigma_{1}, \sigma_{2}\right)\right| f d \nu,
$$

with some $C=C_{c_{2}}>0$.

Proof: By the definition of $\omega_{x}$, denoting $y=x+e_{j}$, we have

$$
\begin{aligned}
\int h_{x}^{\ell, j}\left(\omega_{2, y}-\omega_{2, x}\right) f d \nu= & \int h_{x}^{\ell, j}\left(\frac{\sigma_{2, y}}{\chi\left(u_{2}(y)\right)}-\frac{\sigma_{2, x}}{\chi\left(u_{2}(x)\right)}\right) f d \nu \\
& \quad-\int h_{x}^{\ell, j}\left(\frac{u_{2}(y)}{\chi\left(u_{2}(y)\right)}-\frac{u_{2}(x)}{\chi\left(u_{2}(x)\right)}\right) f d \nu \\
= & I_{1}-I_{2} .
\end{aligned}
$$

For $I_{2}$, we have

$$
\begin{aligned}
& \left|\frac{u_{2}(y)}{\chi\left(u_{2}(y)\right)}-\frac{u_{2}(x)}{\chi\left(u_{2}(x)\right)}\right| \\
& \leq \frac{1}{\chi\left(u_{2}(x)\right) \chi\left(u_{2}(y)\right)}\left\{\chi\left(u_{2}(x)\right)\left|u_{2}(y)-u_{2}(x)\right|+\left|u_{2}(x)\right|\left|\chi\left(u_{2}(x)\right)-\chi\left(u_{2}(y)\right)\right|\right\} \\
& \leq C e^{c_{1} K}\left|\nabla_{x, y}^{1} u_{2}\right| .
\end{aligned}
$$

On the other hand, $I_{1}$ can be rewritten as

$$
\begin{aligned}
I_{1} & =\int \frac{h_{x}^{\ell, j}}{\chi\left(u_{2}(x)\right)}\left(\sigma_{2, y}-\sigma_{2, x}\right) f d \nu+\int h_{x}^{\ell, j}\left(\frac{1}{\chi\left(u_{2}(y)\right)}-\frac{1}{\chi\left(u_{2}(x)\right)}\right) \sigma_{2, y} f d \nu \\
& =: I_{1,1}+I_{1,2} .
\end{aligned}
$$

For $I_{1,1}$, recalling the invariance of $h_{x}^{\ell, j}$, one can apply Lemma 3.4 and obtain

$$
I_{1,1}=\frac{1}{\chi\left(u_{2}(x)\right)} \int h_{x}^{\ell, j} \sigma_{2, x}\left(f\left(\sigma_{1}, \sigma_{2}^{x, y}\right)-f\left(\sigma_{1}, \sigma_{2}\right)\right) d \nu+\frac{1}{\chi\left(u_{2}(x)\right)} R_{1} .
$$

Finally for $I_{1,2}$,

$$
\left|\frac{1}{\chi\left(u_{2}(y)\right)}-\frac{1}{\chi\left(u_{2}(x)\right)}\right|=\frac{\left|\chi\left(u_{2}(x)\right)-\chi\left(u_{2}(y)\right)\right|}{\chi\left(u_{2}(x)\right) \chi\left(u_{2}(y)\right)} \leq C e^{2 c_{1} K}\left|\nabla_{x, y}^{1} u_{2}\right| .
$$

Therefore, we obtain the conclusion.

We can estimate the first term in the right hand side of (3.5) with $y=x+e_{j}$ by the Dirichlet form and obtain

Lemma 3.6. Let $\nu=\nu_{u_{1}(\cdot), u_{2}(\cdot)}$ be the Bernoulli measure satisfying the same condition as in Lemma 3.4. Then, for every $\beta>0$, we have

$$
\left|\int h_{x}^{\ell, j}\left(\omega_{2, x+e_{j}}-\omega_{2, x}\right) f d \nu\right| \leq \beta \mathcal{D}_{x, x+e_{j}}(\sqrt{f} ; \nu)+\frac{C}{\beta} e^{3 c_{1} K} \int\left(h_{x}^{\ell, j}\right)^{2} f d \nu+R_{2, x, j},
$$


where $\mathcal{D}_{x, x+e_{j}}(\sqrt{f} ; \nu)$ is a piece of $\mathcal{D}(\sqrt{f} ; \nu)$ defined on the bond $\left\{x, x+e_{j}\right\}$ and $R_{2, x, j}$ has a bound (3.6).

Proof: For simplicity, we write $y$ for $x+e_{j}$. By decomposing $f\left(\sigma_{1}, \sigma_{2}^{x, y}\right)-f\left(\sigma_{1}, \sigma_{2}\right)=$ $\left(\sqrt{f\left(\sigma_{1}, \sigma_{2}^{x, y}\right)}+\sqrt{f\left(\sigma_{1}, \sigma_{2}\right)}\right)\left(\sqrt{f\left(\sigma_{1}, \sigma_{2}^{x, y}\right)}-\sqrt{f\left(\sigma_{1}, \sigma_{2}\right)}\right)$, the modulus of the first term in the right hand side of (3.5) can be bounded from above by

$$
\beta \mathcal{D}_{x, y}(\sqrt{f} ; \nu)+\frac{C}{\beta \chi\left(u_{2}(x)\right)^{2}} \int\left(h_{x}^{\ell, j}\right)^{2}\left\{f\left(\sigma_{1}, \sigma_{2}^{x, y}\right)+f\left(\sigma_{1}, \sigma_{2}\right)\right\} d \nu
$$

The integral in the second term divided by $\chi\left(u_{2}(x)\right)^{2}$ is equal to and bounded by

$$
\begin{aligned}
\frac{1}{\chi\left(u_{2}(x)\right)^{2}} & \int\left(h_{x}^{\ell, j}\right)^{2} f\left(\sigma_{1}, \sigma_{2}\right)\left(1+r_{x y}\left(\sigma_{2}\right)\right) d \nu \\
& \leq \frac{1+C_{0} e^{c_{1} K}\left|\nabla_{x, y}^{1} u_{2}\right|}{\chi\left(u_{2}(x)\right)^{2}} \int\left(h_{x}^{\ell, j}\right)^{2} f d \nu \\
& \leq e^{2 c_{1} K}\left(1+C_{0} e^{c_{1} K}\left|\nabla_{x, y}^{1} u_{2}\right|\right) \int\left(h_{x}^{\ell, j}\right)^{2} f d \nu
\end{aligned}
$$

This shows the conclusion by recalling $\left|\nabla_{x, y}^{1} u_{2}\right| \leq 2 c_{2}$.

Proof of Proposition 3.1: Recalling (3.3) and by Lemma 3.6 taking $\beta=\frac{\alpha N^{2}}{K}$ with $\alpha>0$ sufficiently small, we have

$$
\begin{aligned}
& \int\left(V-V^{\ell}\right) f d \nu=K \sum_{j=1}^{d} \sum_{x \in \mathbb{T}_{N}^{d}} \int h_{x}^{\ell, j}\left(\omega_{2, x+e_{j}}-\omega_{2, x}\right) f d \nu \\
& \quad \leq \alpha N^{2} \mathcal{D}(\sqrt{f} ; \nu)+\frac{C K^{2}}{\alpha N^{2}} e^{3 c_{1} K} \sum_{j=1}^{d} \sum_{x \in \mathbb{T}_{N}^{d}} \int\left(h_{x}^{\ell, j}\right)^{2} f d \nu+K \sum_{j=1}^{d} \sum_{x \in \mathbb{T}_{N}^{d}} R_{2, x, j}
\end{aligned}
$$

For $R_{2, x, j}$, since $\left|\nabla_{x, x+e_{j}}^{1} u_{2}\right| \leq \frac{C K}{N}$ from Proposition 2.7, by (3.6) estimating $\left|h_{x}^{\ell, j}\right| \leq 1+\left(h_{x}^{\ell, j}\right)^{2}$, we have

$$
K\left|R_{2, x, j}\right| \leq \frac{C K^{2}}{N} e^{3 c_{1} K} \int\left(1+\left(h_{x}^{\ell, j}\right)^{2}\right) f d \nu
$$

Thus, we obtain

$$
\begin{aligned}
\int\left(V-V^{\ell}\right) f d \nu \leq & \alpha N^{2} \mathcal{D}(\sqrt{f} ; \nu) \\
& +\frac{C_{\alpha} K^{2}}{N} e^{3 c_{1} K} \sum_{j=1}^{d} \sum_{x \in \mathbb{T}_{N}^{d}} \int\left(h_{x}^{\ell, j}\right)^{2} f d \nu+C K^{2} e^{3 c_{1} K} N^{d-1}
\end{aligned}
$$


For the second term, we first decompose the sum $\sum_{x \in \mathbb{T}_{N}^{d}}$ as $\sum_{y \in \Lambda_{2 \ell}} \sum_{z \in(4 \ell) \mathbb{T}_{N}^{d}}$ and apply the entropy inequality noting that the variables $\left\{h_{x}^{\ell, j}\right\}$ are $(2 \ell-1)$ dependent:

$$
\begin{aligned}
\sum_{x \in \mathbb{T}_{N}^{d}} \int\left(h_{x}^{\ell, j}\right)^{2} f d \nu & \leq \frac{1}{\gamma} \sum_{y \in \Lambda_{2 \ell}}\left(H(\mu \mid \nu)+\log \int \exp \left\{\gamma \sum_{z \in(4 \ell) \mathbb{T}_{N}^{d}}\left(h_{z+y}^{\ell, j}\right)^{2}\right\} d \nu\right) \\
& =\frac{1}{\gamma}(4 \ell)^{d}\left(H(\mu \mid \nu)+\sum_{z \in(4 \ell) \mathbb{T}_{N}^{d}} \log \int \exp \left\{\gamma\left(h_{z+y}^{\ell, j}\right)^{2}\right\} d \nu\right)
\end{aligned}
$$

However, since $h_{x}^{\ell, j}$ is a weighted sum of independent random variables, by applying Lemma 3.7 (concentration inequality) stated below, we have

$$
\log \int e^{\gamma\left(h_{x}^{\ell, j}\right)^{2}} d \nu \leq 2
$$

for every $0<\gamma \leq \frac{C_{0}}{\sigma^{2}}$, where $C_{0}$ is a universal constant and $\sigma^{2}$ is the supremum of the variances of $h_{x}^{\ell, j}$. By Lemma 3.2,

$$
\sigma^{2} \leq C_{d} g_{d}(\ell)
$$

Therefore, we have

$$
\sum_{x \in \mathbb{T}_{N}^{d}} \int\left(h_{x}^{\ell, j}\right)^{2} f d \nu \leq \frac{1}{\gamma}(4 \ell)^{d}\left(H(\mu \mid \nu)+2\left(\frac{N}{4 \ell}\right)^{d}\right) .
$$

Thus, choosing $\frac{1}{\gamma}=\frac{C_{d}}{C_{0}} g_{d}(\ell)$, we have shown

$$
\begin{aligned}
\int\left(V-V^{\ell}\right) f d \nu \leq & \alpha N^{2} \mathcal{D}(\sqrt{f} ; \nu)+\frac{\bar{C}_{\alpha} \ell^{d} g_{d}(\ell) K^{2} e^{3 c_{1} K}}{N}\left(H(\mu \mid \nu)+\frac{N^{d}}{\ell^{d}}\right) \\
& +C K^{2} e^{3 c_{1} K} N^{d-1} .
\end{aligned}
$$

Now recall $1 \leq K \leq \delta(\log N)^{1 / 2} \leq \delta \log N$ so that $e^{3 c_{1} K} \leq N^{3 c_{1} \delta}$ and choose $\delta>0$ such that $3 c_{1} \delta \leq \kappa$ for a given small $\kappa>0$. Choose $\ell=N^{\frac{1}{d}-\kappa}$ when $d \geq 2$ and $N^{\frac{1}{2}-\kappa}$ when $d=1$. Then, when $d \geq 2$, we have

$$
\begin{aligned}
& \frac{\ell^{d} g_{d}(\ell) K^{2}}{N} e^{3 c_{1} K} \leq C N^{-\kappa(d-1)}(\log N)^{3} \leq 1, \quad \frac{N^{d}}{\ell^{d}}=N^{d-1+d \kappa}, \\
& K^{2} e^{3 c_{1} K} N^{d-1} \leq N^{d-1+2 \kappa},
\end{aligned}
$$

which shows (3.2). When $d=1$,

$$
\frac{\ell^{2} K^{2}}{N} e^{3 c_{1} K} \leq \delta^{2} N^{-\kappa} \log N \leq 1, \quad \frac{N}{\ell}=N^{\frac{1}{2}+\kappa}, \quad K^{2} e^{3 c_{1} K} N^{d-1} \leq N^{2 \kappa} .
$$

This shows the conclusion for $d=1$.

Lemma 3.7. (concentration inequality) Let $\left\{X_{i}\right\}_{i=1}^{n}$ be independent random variables with values in the intervals $\left[a_{i}, b_{i}\right]$. Set $\bar{X}_{i}=X_{i}-E\left[X_{i}\right]$ and $\kappa=\sum_{i=1}^{n}\left(b_{i}-\right.$ $\left.a_{i}\right)^{2}$. Then, for every $\gamma \in\left[0, \kappa^{-1}\right]$, we have

$$
\log E\left[\exp \left\{\gamma\left(\sum_{i=1}^{n} \bar{X}_{i}\right)^{2}\right\}\right] \leq 2 \gamma \kappa .
$$

The second step is to estimate the integral $\int V^{\ell} f d \nu$, where $V^{\ell}$ is given by (3.1). 
Proposition 3.8. We assume the same conditions as Proposition 3.1. Then, for $\kappa>0$, we have

$$
\int V^{\ell} f d \nu \leq C K H\left(\mu_{t} \mid \nu_{t}\right)+C_{\kappa} N^{d-1+\kappa},
$$

with some $C_{\kappa}>0$ when $d \geq 2$. When $d=1$, the last term is replaced by $C_{\kappa} N^{\frac{1}{2}+\kappa}$.

Proof: We again decompose the sum $\sum_{x \in \mathbb{T}_{N}^{d}}$ in (3.1) as $\sum_{y \in \Lambda_{2 \ell}} \sum_{z \in(4 \ell) \mathbb{T}_{N}^{d}}$, and

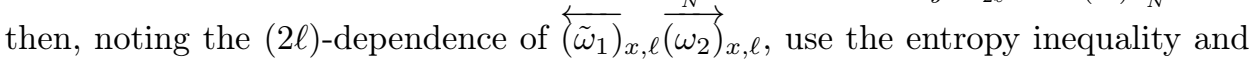
the concentration inequality to show

$$
\begin{aligned}
\int V^{\ell} f d \nu & \leq \frac{K}{\gamma} \sum_{y \in \Lambda_{2 \ell}}\left\{H\left(\mu_{t} \mid \nu_{t}\right)+\sum_{z \in(4 \ell) \mathbb{T}_{N}^{d}} \log E^{\nu t}\left[e^{\gamma\left(\tilde{\tilde{\omega}}_{1}\right)_{z+y, \ell}\left(\overrightarrow{(\omega)}_{z+y, \ell}\right]}\right\}\right. \\
& \leq \frac{K(4 \ell)^{d}}{\gamma}\left\{H\left(\mu_{t} \mid \nu_{t}\right)+\frac{N^{d}}{(4 \ell)^{d}} C_{1} \gamma \ell^{-d}\right\}
\end{aligned}
$$

for $\gamma=c \ell^{d}$ with $c>0$ small enough. Note that, by the central limit theorem,

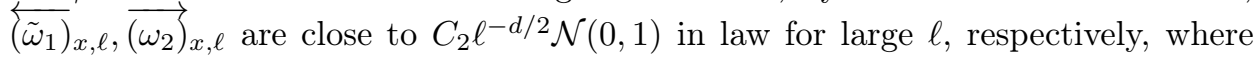
$\mathcal{N}(0,1)$ denotes the standard Gaussian. Since $\ell=N^{\frac{1}{d}-\kappa}$ when $d \geq 2$, we have $\frac{K N^{d}}{\ell^{d}} \leq N^{d-1+d \kappa} \cdot \delta \log N$ and obtain (3.7). When $d=1$, since $\ell=N^{\frac{1}{2}-\kappa}$, we have $\frac{K N^{d}}{\ell^{d}} \leq N^{\frac{1}{2}+\kappa} \delta \log N$.

3.2. Proof of (2.9). We now discuss the contribution of

$$
V_{1}:=-\frac{N^{2}}{2} \sum_{x, y \in \mathbb{T}_{N}^{d}:|x-y|=1}\left(u_{1}(y)-u_{1}(x)\right)^{2} \omega_{1, x} \omega_{1, y}
$$

in (2.5), which arises from the Kawasaki part; similar computations are made for $V_{1}$ in Funaki and Tsunoda (2018). The second term $V_{2}$ can be treated similarly. We may think $N^{2}\left(u_{1}(y)-u_{1}(x)\right)^{2}$ as if $K$ in the argument we have developed. However, from Proposition 2.7, we have

$$
N^{2}\left(u_{1}(y)-u_{1}(x)\right)^{2} \leq C K^{2} .
$$

This means that we may replace $K$ by $K^{2}$ properly in the estimates obtained in Propositions 3.1 and 3.8 for the first and second terms. Since $K^{2} \leq \delta^{2} \log N$ appearing in the error terms can be absorbed by $N^{\kappa}$ for every $\kappa>0$, this leads to

$$
\int\left(V_{1}+V_{2}\right) d \mu_{t} \leq \alpha N^{2} \mathcal{D}\left(\sqrt{\frac{d \mu_{t}}{d \nu_{t}}} ; \nu_{t}\right)+C_{\kappa} K^{2} H\left(\mu_{t} \mid \nu_{t}\right)+C_{\alpha, \kappa} N^{d-1+\kappa},
$$

for every $a, \kappa>0$, when $d \geq 2$ and the last term is replaced by $C_{\alpha, \kappa} N^{\frac{1}{2}+\kappa}$ when $d=1$.

\section{Consequence of Theorem 2.2}

Recall that $\mu_{t}^{N}$ is the distribution of $\tilde{\sigma}^{N}(t)$ on $\mathcal{X}_{N}^{2}$ and $u_{i}^{N}(t)=\left\{u_{i}^{N}(t, x)\right\}_{x \in \mathbb{T}_{N}^{d}}$, $i=1,2$ is the solution of the discretized hydrodynamic equation (2.3). The 
Bernoulli measure on $\mathcal{X}_{N}^{2}$ with mean $\left\{u_{i}^{N}(t, x)\right\}_{x \in \mathbb{T}_{N}^{d}}$ is denoted by $\nu_{t}^{N}$. Then Theorem 2.2 shows $H\left(\mu_{t}^{N} \mid \nu_{t}^{N}\right)=o\left(N^{d}\right)$ under a proper choice of $K=K(N) \nearrow \infty$. We define macroscopic functions $u_{i}^{N}(t, r), r \in \mathbb{T}^{d}$ as step functions

$$
u_{i}^{N}(t, r)=\sum_{x \in \mathbb{T}_{N}^{d}} u_{i}^{N}(t, x) 1_{B\left(\frac{x}{N}, \frac{1}{N}\right)}(r), \quad r \in \mathbb{T}^{d},
$$

from the microscopic functions $u_{i}^{N}(t, x), x \in \mathbb{T}_{N}^{d}$, where $B\left(\frac{x}{N}, \frac{1}{N}\right)=\prod_{j=1}^{d}\left[\frac{x_{j}}{N}-\right.$ $\left.\frac{1}{2 N}, \frac{x_{j}}{N}+\frac{1}{2 N}\right)$ is the box with center $\frac{x}{N}$ and side length $\frac{1}{N}$.

Under our choice of $K$, the entropy inequality

$$
\mu_{t}^{N}(A) \leq \frac{\log 2+H\left(\mu_{t}^{N} \mid \nu_{t}^{N}\right)}{\log \left\{1+1 / \nu_{t}^{N}(A)\right\}}
$$

combined with Proposition 4.1 stated below shows that

$$
\lim _{N \rightarrow \infty} \mu_{t}^{N}\left(\mathcal{A}_{N, t}^{\varepsilon}\right)=0
$$

for every $\varepsilon>0$, where

$\mathcal{A}_{N, t}^{\varepsilon} \equiv \mathcal{A}_{N, t, \varphi}^{\varepsilon}:=\left\{\tilde{\sigma} \in \mathcal{X}_{N}^{2} ;\left|\left\langle\alpha_{i}^{N}, \varphi\right\rangle-\left\langle u_{i}^{N}(t, \cdot), \varphi\right\rangle\right|>\varepsilon, i=1,2\right\}, \varphi \in C^{\infty}\left(\mathbb{T}^{d}\right)$.

Proposition 4.1. There exists $C=C_{\varepsilon, \varphi}>0$ such that

$$
\nu_{t}^{N}\left(\mathcal{A}_{N, t}^{\varepsilon}\right) \leq e^{-C N^{d}} .
$$

Proof: Since

$$
X_{i}:=\left\langle\alpha_{i}^{N}, \varphi\right\rangle-\left\langle u_{i}^{N}(t, \cdot), \varphi\right\rangle=\frac{1}{N^{d}} \sum_{x \in \mathbb{T}_{N}^{d}}\left\{\sigma_{i, x}-u_{i}^{N}\left(t, \frac{x}{N}\right)\right\} \varphi\left(\frac{x}{N}\right)+o(1),
$$

for $\varphi \in C^{\infty}\left(\mathbb{T}^{d}\right)$, we have

$$
\begin{aligned}
\nu_{t}^{N}\left(\mathcal{A}_{N, t}^{\varepsilon}\right) & \leq e^{-\gamma \varepsilon N^{d}} E^{\nu_{t}^{N}}\left[e^{\gamma N^{d}\left|X_{i}\right|}\right] \\
& \leq e^{-\gamma \varepsilon N^{d}}\left\{E^{\nu_{t}^{N}}\left[e^{\gamma N^{d} X_{i}}\right]+E^{\nu_{t}^{N}}\left[e^{-\gamma N^{d} X_{i}}\right]\right\},
\end{aligned}
$$

for every $\gamma>0$. However, by the independence of $\sigma_{i, x}$ under $\nu_{t}^{N}$, we have

$$
\begin{aligned}
E^{\nu_{t}^{N}}\left[e^{ \pm \gamma N^{d} X_{i}}\right] & =\prod_{x \in \mathbb{T}_{N}^{d}} E^{\nu_{t}^{N}}\left[e^{ \pm \gamma\left\{\sigma_{i, x}-u_{i, x}\right\} \varphi_{x}+o(1)}\right] \\
& =\prod_{x \in \mathbb{T}_{N}^{d}}\left\{e^{ \pm \gamma\left(1-u_{i, x}\right) \varphi_{x}} u_{i, x}+e^{\mp \gamma u_{i, x} \varphi_{x}}\left(1-u_{i, x}\right)\right\}+o(1),
\end{aligned}
$$

where $u_{i, x}=u_{i}^{N}(t, x)$ and $\varphi_{x}=\varphi\left(\frac{x}{N}\right)$. However, by the Taylor's formula applied at $\gamma=0$, we see

$$
\left|e^{ \pm \gamma\left(1-u_{i, x}\right) \varphi_{x}} u_{i, x}+e^{\mp \gamma u_{i, x} \varphi_{x}}\left(1-u_{i, x}\right)-1\right| \leq \frac{\gamma^{2}}{2} C, \quad C=C_{\|\varphi\|_{\infty}},
$$

for $0<\gamma \leq 1$. Thus we obtain

$$
\nu_{t}^{N}\left(\mathcal{A}_{N, t}^{\varepsilon}\right) \leq e^{-\gamma \varepsilon N^{d}+C \gamma^{2} N^{d}},
$$

for $\gamma>0$ sufficiently small. This shows the conclusion. 


\section{Convergence of the solution of the discretized hydrodynamic equation to that of the free boundary problem}

We show $u_{i}^{N}(t, r), t \in[0, T], r \in \mathbb{T}^{d}, i=1,2$ appearing in (4.2), which is defined by (4.1) from the solution of the discretized hydrodynamic equation (2.3), converges to the unique weak solution of the free boundary problem (1.3). This can be done along with Crooks et al. (2004), in a discrete setting. Once this is shown, combined with (4.2), the proof of Theorem 1.1 is complete.

\section{Lemma 5.1.}

$$
\int_{0}^{T} \int_{\mathbb{T}^{d}} u_{1}^{N}(t, r) u_{2}^{N}(t, r) d t d r \leq \frac{1}{K} .
$$

Proof: (cf. Lemma 2.3 of Crooks et al., 2004 with $\varphi \equiv 1$ ) From (2.3), we have

$$
\begin{aligned}
K & \sum_{x \in \mathbb{T}_{N}^{d}} \int_{0}^{T} u_{1}^{N}(t, x) u_{2}^{N}(t, x) d t \\
& =d_{1} \sum_{x \in \mathbb{T}_{N}^{d}} \int_{0}^{T} \Delta^{N} u_{1}^{N}(t, x) d t+\sum_{x \in \mathbb{T}_{N}^{d}} u_{1}^{N}(0, x)-\sum_{x \in \mathbb{T}_{N}^{d}} u_{1}^{N}(T, x) \leq N^{d},
\end{aligned}
$$

which implies the conclusion.

\section{Lemma 5.2.}

$$
\int_{0}^{T} \int_{\mathbb{T}^{d}}\left|\nabla^{N} u_{i}^{N}(t, r)\right|^{2} d t d r \leq \frac{1}{2 d_{i}}, \quad i=1,2
$$

where $\nabla^{N} u(r)=\left\{N\left(u\left(r+\frac{1}{N} e_{j}\right)-u(r)\right)\right\}_{j=1}^{d}$.

Proof: (cf. Lemma 2.4 of Crooks et al., 2004 with $\varphi \equiv 1$ ) From (2.3), we have

$$
\frac{1}{2} \frac{d}{d t} \int_{\mathbb{T}^{d}} u_{1}^{N}(t, r)^{2} d r+d_{1} \int_{\mathbb{T}^{d}}\left|\nabla^{N} u_{1}^{N}(t, r)\right|^{2} d r=-K \int_{\mathbb{T}^{d}} u_{1}^{N}(t, r)^{2} u_{2}^{N}(t, r) d r \leq 0,
$$

and this implies

$$
d_{1} \int_{0}^{T} d t \int_{\mathbb{T}^{d}}\left|\nabla^{N} u_{1}^{N}(t, r)\right|^{2} d r \leq \frac{1}{2} \int_{\mathbb{T}^{d}}\left\{u_{1}^{N}(0, r)^{2}-u_{1}^{N}(T, r)^{2}\right\} d r \leq \frac{1}{2} .
$$

The proof for $u_{2}^{N}$ is similar.

These two lemmas with the help of Fréchet-Kolmogorov theorem show that $\left\{u_{i}^{N}(t, r)\right\}_{N}$ are relatively compact in $L^{2}\left([0, T] \times \mathbb{T}^{d}\right)$. In fact, two lemmas prove the equi-continuity of $\left\{u_{i}^{N}(t, r)\right\}_{N}$ in the space $L^{2}\left([0, T] \times \mathbb{T}^{d}\right)$ as in Lemmas 2.6 and 2.7 of Crooks et al. (2004).

Corollary 5.3. (cf. Corollary 3.1 of Crooks et al., 2004) From any subsequence of $\left\{u_{i}^{N}(t, r)\right\}_{N}, i=1,2$, one can find further subsequences $\left\{u_{i}^{N_{k}}(t, r)\right\}_{k}, i=1,2$, and $u_{i} \in L^{2}\left([0, T] \times \mathbb{T}^{d}\right), i=1,2$ such that

$$
u_{i}^{N_{k}} \rightarrow u_{i} \quad \text { strongly in } L^{2}\left([0, T] \times \mathbb{T}^{d}\right) \text { and a.e. in }[0, T] \times \mathbb{T}^{d}
$$

as $k \rightarrow \infty$. 
Lemma 5.4. (cf. Lemma 3.2 of Crooks et al., 2004) $u_{1} u_{2}=0$ a.e. in $[0, T] \times \mathbb{T}^{d}$. Set

$$
w^{N}:=u_{1}^{N}-u_{2}^{N} \quad \text { and } \quad w:=u_{1}-u_{2} .
$$

From Corollary 5.3 and Lemma $5.4, w^{N_{k}} \rightarrow w$ strongly in $L^{2}\left([0, T] \times \mathbb{T}^{d}\right)$ and a.e. in $[0, T] \times \mathbb{T}^{d}$ as $k \rightarrow \infty$ and furthermore

$$
u_{1}=w^{+} \quad \text { and } \quad u_{2}=w^{-} .
$$

Proposition 5.5. $w$ is the unique weak solution of (1.3).

Proof: It is sufficient to check the property (ii) of Definition 1.2 for $w$. From (2.3), for $\psi \in C^{1,2}\left([0, T] \times \mathbb{T}^{d}\right)$ such that $\psi(T, r)=0$ for all $r$,

$$
\begin{gathered}
\int_{0}^{T} \int_{\mathbb{T}^{d}}\left(u_{1}^{N}(t, r)-u_{2}^{N}(t, r)\right) \partial_{t} \psi(t, r) d r d t-\int_{\mathbb{T}^{d}}\left(u_{1}^{N}(0, r)-u_{2}^{N}(0, r)\right) \psi(0, r) d r \\
=\int_{0}^{T} \int_{\mathbb{T}^{d}}\left(d_{1} u_{1}^{N}(t, r)-d_{2} u_{2}^{N}(t, r)\right) \Delta^{N} \psi(t, r) d r d t
\end{gathered}
$$

We obtain the property (ii) for $w$ by passing to the limit $k \rightarrow \infty$ along with the subsequence $N=N_{k}$.

Because of the uniqueness of $w$, without taking subsequences, $u_{i}^{N}(t, r), i=1,2$ themselves converge to $u_{i}(t, r)$ strongly in $L^{2}\left([0, T] \times \mathbb{T}^{d}\right)$ and a.e. in $[0, T] \times \mathbb{T}^{d}$ as $N \rightarrow \infty$. This combined with (4.2) completes the proof of Theorem 1.1.

Acknowledgments: T. Funaki is supported in part by JSPS KAKENHI, Grantin-Aid for Scientific Researches (A) 18H03672 and (S) 16H06338. E. Presutti thanks the GSSI. M. E. Vares acknowledges support of CNPq (grant 305075/2016-0) and FAPERJ (grant E-26/203.048/2016).

\section{References}

E. C. M. Crooks, E. N. Dancer, D. Hilhorst, M. Mimura and H. Ninomiya. Spatial segregation limit of a competition-diffusion system with Dirichlet boundary conditions. Nonlinear Anal. Real World Appl. 5 (4), 645-665 (2004). MR2079274.

E. N. Dancer, D. Hilhorst, M. Mimura and L. A. Peletier. Spatial segregation limit of a competition-diffusion system. European J. Appl. Math. 10 (2), 97-115 (1999). MR1687440.

E. Daus, L. Desvillettes and A. Jüngel. Cross-diffusion systems and fast-reaction limits. ArXiv Mathematics e-prints (2017). arXiv: 1710.03590.

T. Delmotte and J.-D. Deuschel. On estimating the derivatives of symmetric diffusions in stationary random environment, with applications to $\nabla \phi$ interface model. Probab. Theory Related Fields 133 (3), 358-390 (2005). MR2198017.

T. Funaki. Free boundary problem from stochastic lattice gas model. Ann. Inst. H. Poincaré Probab. Statist. 35 (5), 573-603 (1999). MR1705681.

T. Funaki. Hydrodynamic limit for exclusion processes. Commun. Math. Stat. 6 (4), 417-480 (2018). MR3877713.

T. Funaki and K. Tsunoda. Motion by mean curvature from Glauber-Kawasaki dynamics. ArXiv Mathematics e-prints (2018). arXiv: 1812.10182.

M. Iida, H. Monobe, H. Murakawa and H. Ninomiya. Vanishing, moving and immovable interfaces in fast reaction limits. J. Differential Equations 263 (5), 2715-2735 (2017). MR3655798. 
M. Jara and O. Menezes. Non-equilibrium fluctuations of interacting particle systems. ArXiv Mathematics e-prints (2018). arXiv: 1810.09526.

M. Sasada. Hydrodynamic limit for two-species exclusion processes. Stochastic Process. Appl. 120 (4), 494-521 (2010). MR2594368.

D. W. Stroock and W. Zheng. Markov chain approximations to symmetric diffusions. Ann. Inst. H. Poincaré Probab. Statist. 33 (5), 619-649 (1997). MR1473568. 